\title{
Effective QED actions: Representations, gauge invariance, anomalies, and mass expansions
}

\author{
S. Deser* \\ Department of Physics, Brandeis University, Waltham, Massachusetts 02254 \\ L. Griguolo ${ }^{\dagger}$ \\ Center for Theoretical Physics, Laboratory for Nuclear Science and Department of Physics, Massachusetts Institute of Technology, \\ Cambridge, Massachusetts 02139 \\ D. Seminara \\ Department of Physics, Brandeis University, Waltham, Massachusetts 02254 \\ (Received 8 December 1997; published 15 May 1998)
}

\begin{abstract}
We analyze and give explicit representations for the effective Abelian vector gauge field actions generated by charged fermions with particular attention to the thermal regime in odd dimensions, where spectral asymmetry can be present. We show, through $\zeta$-function regularization, that both small and large gauge invariances are preserved at any temperature and for any number of fermions at the usual price of anomalies: helicity (parity) invariance will be lost in even (odd) dimensions, and in the latter even at zero mass. Gauge invariance dictates a very general "Fourier" representation of the action in terms of the holonomies that carry the novel, large gauge-invariant, information. We show that large (unlike small) transformations and hence their Ward identities are not perturbative order-preserving, and clarify the role of (properly redefined) Chern-Simons terms in this context. From a powerful representation of the action in terms of massless heat kernels, we are able to obtain rigorous gauge-invariant expansions, for both small and large fermion masses, of its separate parity even and odd parts in arbitrary dimension. The representation also displays both the nonperturbative origin of a finite renormalization ambiguity and its physical resolution by requiring decoupling at infinite mass. Finally, we illustrate these general results by explicit computation of the effective action for some physical examples of field configurations in the three-dimensional case, where our conclusions on finite temperature effects may have physical relevance. Non-Abelian results will be presented separately. [S0556-2821(98)01012-1]
\end{abstract}

PACS number(s): 11.10.Wx, 11.15.Bt, 11.30.Er, 11.30.Rd

\section{INTRODUCTION}

Effective gauge field actions, induced by integrating out their sources, play an essential role in physics. Here we will study the result of integrating out charged fermions minimally coupled to an Abelian vector potential, with emphasis on odd dimensions, especially $D=3$, and on the thermal regime in which topological considerations are both essential and delicate. The corresponding non-Abelian analysis will be presented subsequently [1]. Three-dimensional QED $\left(\mathrm{QED}_{3}\right)$ models are interesting for a number of reasons: From a theoretical point of view they provide fascinating examples of interrelations between quantum mechanics, unusual gauge invariance, topology, and discrete space-time symmetries $[2,3]$. On more physical grounds, they are natural candidates for the description of planar phenomena in the condensed matter context [4] or the high-temperature regime of fourdimensional models [5].

Many intriguing features of odd-dimensional dynamics stem from the existence of the unconventional, parityviolating, but apparently gauge invariant and well-defined Chern-Simons (CS) term, which has its simplest, quadratic, form in the planar $(d=3)$ case [3]:

\footnotetext{
*Email address: Deser@binah.cc.brandeis.edu

†Email address: Griguolo@irene.mit.edu

†Email address: Seminara@binah.cc.brandeis.edu
}

$$
I_{\mathrm{CS}}=\int d^{3} x \epsilon^{\lambda \mu \nu} A_{\lambda} F_{\mu \nu}
$$

However, as we shall see, $I_{\mathrm{CS}}$ is neither gauge invariant (which is in fact one essential reason for its interest) nor is it generically well defined, but it can be "improved"; it also does not appear "unaccompanied" in the effective actions. Understanding these points plays a pivotal role both in analyzing $\mathrm{QED}_{3}$, as well as incorporating correctly possible "bare" CS terms that could be present in a descent from the $D=4$ QED topological action "theta" term $\int F \wedge F$. In this connection, one must also come to terms with the proper quantization requirements, stemming from their gauge dependence, on the coefficients of $I_{\mathrm{CS}}$. We will deal with $I_{\mathrm{CS}}$ in Sec. II, as part of a general analysis of the complete effective actions and their gauge properties, extending work begun in Ref. [6]. We will then review why the perturbative expansion of the effective action in the coupling constant is not invariant under large (not contractable to the identity) gauge transformations, thereby invalidating the usual perturbative Ward identity counting. Analyzing how gauge invariance constrains the form of the full effective action in terms of its dependence on the variables carrying the local and global degrees of freedom, namely the field strength and the holonomy, will bring in the CS term (in its correct, "improved" guise) as the carrier of global information.

In Sec. III, we shall detail the properties of the Dirac determinant in the rigorous framework of $\zeta$-function regular- 
ization with particular attention to the delicate interplay between large gauge invariance and spectral asymmetry. This analysis will make manifest the necessary clash in odd (even) dimensions between parity (helicity) and gauge invariance. In odd dimension an "intrinsic" parity anomaly, i.e., one nonvanishing even for massless (hence formally parity conserving) fermions, is generally present and it is identified with the $\eta$ function, more precisely with $\eta(0)$ [7]. This quantity will be seen to be a discontinuous gauge invariant functional of the fields, containing, as a single unit, the CS action together with a nonlocal object given by the index; while the former can be easily recovered in a perturbative approach, the latter, being discontinuous, becomes manifest only through a nonperturbative investigation of the Dirac determinant. We shall also notice that the parity-violating part of the effective action suffers from a sign ambiguity whose mathematical origin stems from having to specify a choice of cut in the definition of the complex power of the Dirac eigenvalues; physically, this is a finite regularization effect which has its counterpart also in the perturbative regime. The ambiguity can be fixed by requiring a vanishing effective action, i.e., "decoupling," in the infinite fermion mass limit. We then obtain an explicit "spectral" representation of the action in terms of massless heat kernels.

In Sec. IV, we use this representation to derive systematic (gauge-invariant) mass-expansions in both small and large mass regimes. These expansions, valid for any dimensions, may have a wider applicability and so are given in some detail.

In Sec. V, many of the general features encountered in the previous sections are illustrated by explicit integration in presence of some specific, physically nontrivial, gauge field configurations. This also provides a useful check of the more formal results developed in the earlier sections.

Many results presented here, such as large gauge invariance and mass expansions, can be shown to extend straightforwardly to the non-Abelian context. These, as well some features intrinsic to the non-Abelian case will be discussed in Ref. [1] and also illustrated through explicit configuration examples.

\section{II. $D=3$ : LARGE GAUGE INVARIANCE, EFFECTIVE ACTION, CS TERMS}

In this section we shall focus for concreteness on the important and illustrative case of $d=3$, but much of the discussion is general. Our three-space has $S^{1}$ (time) $\times \Sigma^{2}$ topology, $\Sigma^{2}$ being a compact Riemann two-surface such as a sphere $S^{2}$ or a torus $T^{2}$, depending on the desired spatial boundary conditions. We work with a finite two-volume in order to avoid infrared divergences associated with the continuous spectrum in an open space. Most considerations presented in this section apply naturally to more familiar three-spaces, such as the usual $S^{1} \times \mathbb{R}^{2}$ assumed in the perturbative approach. However, compact $\Sigma^{2}$ allowing for magnetic flux are more physical and will become essential in our full nonperturbative construction below.

The $S^{1}$ circle is identified with euclidean time and its length $\beta=1 / \kappa T$ is the inverse of the temperature. Spinors are required to satisfy antiperiodic boundary conditions

$$
\psi(t+\beta, \mathbf{x})=-\psi(t, \mathbf{x}), \bar{\psi}(t+\beta, \mathbf{x})=-\bar{\psi}(t, \mathbf{x}),
$$

while the $\mathrm{U}(1)$ gauge field is chosen to be periodic,

$$
A_{\mu}(t+\beta, \mathbf{x})=A_{\mu}(t, \mathbf{x}) .
$$

(In the presence of other nontrivial cycles, such as $T^{3}$, one must specify the periodicity conditions also in their characteristic directions.) The fermion action is taken to be

$$
S_{f}=i \int d^{3} x \bar{\psi}(\not D+m) \psi,
$$

where $D_{\mu} \equiv \partial_{\mu}+i A_{\mu}$ is the usual $\mathrm{U}(1)$ covariant derivative, and the $\gamma^{\mu}$ are Hermitian. Requiring the gauge transformations $U$

$$
A_{\mu} \rightarrow A_{\mu}-i U^{-1} \partial_{\mu} U, \quad U \equiv \exp [i \Omega(t, \mathbf{x})],
$$

to respect these periodicities forces them to be periodic as well, but allowing the phase $\Omega$ to obey

$$
\Omega(t+\beta, \mathbf{x})-\Omega(t, \mathbf{x})=2 \pi n, \quad n \in \mathbb{Z} .
$$

Different $n$ in Eq. (2.5) specify gauge transformations belonging to different homotopy classes; only transformations with the same $n$ can be continuously deformed into each other. Those $\Omega(t, \mathbf{x})$ with $n \neq 0$ generate "large" gauge transformations. A representative for each such class can easily be constructed,

$$
U_{n}(t, \mathbf{x})=\exp \left(i \frac{2 \pi}{\beta} n t\right) .
$$

(The composition law $U_{n} \times U_{m}=U_{n+m}$ expresses the mathematical statement that $\Pi_{1}[\mathrm{U}(1)]=\mathrm{Z}$.) Understanding how the invariance under the transformations (2.6) constrains the form of the effective action is a central issue. We begin by showing that the existence of a nontrivial $U_{n}$ invalidates the usual perturbative Ward identity counting. Restoring (for the moment) explicit dependence on the coupling constant $e$, a gauge transformation has the form

$$
A_{\mu} \rightarrow A_{\mu}-\frac{i}{e} U(t, \mathbf{x})^{-1} \partial_{\mu} U(t, \mathbf{x})=A_{\mu} \rightarrow A_{\mu}+\frac{1}{e} \partial_{\mu} \widetilde{\Omega}(t, \mathbf{x}),
$$

with $U(t, \mathbf{x})=\exp [i \widetilde{\Omega}(t, \mathbf{x})]$. If $n=0, \widetilde{\Omega}(t, \mathbf{x})$ is strictly periodic ("small" transformation), and the apparent nonanalytic $1 / e$ behavior in Eq. (2.7) can be made to disappear by redefining $\widetilde{\Omega}(t, \mathbf{x})=e \Omega(t, \mathbf{x})$. Thus a perturbative expansion will be small-invariant order by order because, after the rescaling, Eq. (2.7) cannot mix different orders of perturbation theory. Instead under the large transformations (2.6), the gauge connection changes as follows:

$$
A_{0} \rightarrow A_{0}+\frac{2 \pi}{e} n, \quad A_{i} \rightarrow A_{i} .
$$

A rescaling will merely hide the $1 / e$ factor in the boundary conditions, leaving Eq. (2.8) unaffected. This intrinsic 1/e dependence means that only the full effective action (as we shall show), but not its individual expansion terms (including CS parts) will remain large gauge invariant. In fact the shift in Eq. (2.8) can mix all orders of perturbation theory. (Per- 
turbative noninvariance will also characterize any other expansion that fails to commute with the above boundary condition.)

Let us now see precisely how large gauge invariance restricts the possible structure of the determinant, or indeed of any well-defined gauge field functional. To simplify our argument and avoid irrelevant spatial homotopies, we shall take $\Sigma^{2}$ to be the sphere. Because of the existence of the nontrivial $S^{1}$ cycle, we can construct, besides $F_{\mu \nu}$, a second and independent gauge-invariant object, the holonomy

$$
\begin{aligned}
W(\beta, \mathbf{x}) & \equiv \exp \left(i \int_{0}^{\beta} A_{0}\left(t^{\prime}, \mathbf{x}\right) d t^{\prime}\right) \\
& \equiv \exp \left[i \beta \mathcal{A}_{0}(\mathbf{x})\right] .
\end{aligned}
$$

We will show that $F_{\mu \nu}$ and $W$ together completely specify $A_{\mu}$ up to a gauge. What information carried by $W(\beta, \mathbf{x})$, or equivalently by $\mathcal{A}_{0}$, is not already contained in the field strength? The gradient of $W$ obeys

$$
\begin{gathered}
\nabla W=i W \int_{0}^{\beta} d t^{\prime}\left[\nabla A_{0}\left(t^{\prime}, \mathbf{x}\right)-\partial_{t^{\prime}} \mathbf{A}\left(t^{\prime}, \mathbf{x}\right)\right] \\
=-i W \int_{0}^{\beta} \mathbf{E}\left(t^{\prime}, \mathbf{x}\right) d t^{\prime} \\
E_{i} \equiv F_{0 i}
\end{gathered}
$$

because $\int_{0}^{\beta} d t^{\prime} \partial_{t^{\prime}} \mathbf{A}\left(t^{\prime}, \mathbf{x}\right)$ vanishes by periodicity (2.2); equivalently,

$$
-\nabla \mathcal{A}_{0}(\mathbf{x})=\frac{1}{\beta} \int_{0}^{\beta} d t^{\prime} \mathbf{E}\left(t^{\prime}, \mathbf{x}\right)
$$

Since $W$ is unimodular, the linearity of Eq. (2.10) implies that $W$ is the product of a (generically nonlocal) functional of $\mathbf{E}$ and of the two-geometry times a constant phase factor. The integrability (vanishing curl) of Eq. (2.10) is insured by the Bianchi identity; its general solution is obtained from the divergence of Eq. (2.11) to yield

$$
\mathcal{A}_{0}(\mathbf{x})=\frac{2 \pi}{\beta} a-\int_{0}^{\beta} d t^{\prime} \int d^{2} y G(\mathbf{x}, \mathbf{y}) \nabla \cdot \mathbf{E}\left(t^{\prime}, \mathbf{y}\right) .
$$

Here the Green's function $G(\mathbf{x}, \mathbf{y})$ on the two-sphere obeys $\Delta G(\mathbf{x}, \mathbf{y})=\mathrm{I}-\mathrm{P}$, where $\mathrm{P}$ is the projector on the zero modes. The constant part of $\mathcal{A}_{0}(\mathbf{x})$ corresponds to the constant phase part $\exp (2 \pi i a)$ of $W$, while the rest of $\mathcal{A}_{0}(\mathbf{x})$ exhibits the $\mathbf{E}$ and two-geometry dependence. Thus the new information carried by $W$ is encoded entirely in the topological degree of freedom $a$, the flat connection; ${ }^{1}$ it transforms according to $a \rightarrow a+1$ under large transformations. The fermion determi-

\footnotetext{
${ }^{1}$ The appearance of topological degrees of freedom governing behavior under homotopically nontrivial transformations is not unusual and occurs in other contexts and dimensions. In two dimensions, for example, all the dynamics of Yang-Mills theories is described by such variables.
}

nant can now be viewed as a functional of both $F_{\mu \nu}$ and $a$. Its invariance is assured if the effective action $\Gamma$ obeys the additional finite Ward identity

$$
\Gamma\left(a+1, F_{\mu \nu}\right)=\Gamma\left(a, F_{\mu \nu}\right),
$$

namely, if $\Gamma$ is periodic in $a$; equivalently, Eq. (2.13) expresses the invariance of $\Gamma$ under the Abelian large transformation group $\mathbb{Z}$. The periodicity in $a$ permits us to Fourier expand $\Gamma$ :

$$
\begin{aligned}
\exp \left[-\Gamma\left(F_{\mu \nu}, a\right)\right]= & \sum_{k=-\infty}^{\infty}\left[\hat{\Gamma}_{k}^{(1)}\left(F_{\mu \nu}\right) \cos 2 \pi k a\right. \\
& \left.+\hat{\Gamma}_{k}^{(2)}\left(F_{\mu \nu}\right) \sin 2 \pi k a\right] .
\end{aligned}
$$

Before going further, however, we want to reexpress $a$ in terms of an appropriate functional of the gauge field (but of course not of $F_{\mu \nu}$ alone, as it is insensitive to $a$ ). This is precisely the role of $I_{\mathrm{CS}}$, as defined by Eq. (1.1) (or rather of its corrected version, as we shall see) and we must therefore consider its properties in detail. Under the gauge transformation (2.4), (2.5), we have

$$
I_{\mathrm{CS}}\left(A^{U}\right) \rightarrow I_{\mathrm{CS}}(A)+\int d^{3} x \epsilon^{\lambda \mu \nu} \partial_{\lambda}\left(\Omega F_{\mu \nu}\right)=I_{\mathrm{CS}}(A)+\Delta I_{\mathrm{CS}} .
$$

Although the gauge term in Eq. (2.15) is a total divergence, dropping it is not generally permitted, since

$$
\begin{aligned}
\Delta I_{\mathrm{CS}}= & \int d^{3} x \epsilon^{\lambda \mu \nu} \partial_{\lambda}\left[\Omega(t, \mathbf{x}) F_{\mu \nu}\right] \\
= & 2 \int_{0}^{\beta} d t \partial_{t} \int_{\Sigma} d^{2} x[\Omega(t, \mathbf{x}) B(t, \mathbf{x})] \\
& +2 \int_{0}^{\beta} d t \int_{\Sigma} d^{2} x \epsilon^{i j} \partial_{i}\left[\Omega(t, \mathbf{x}) E_{j}\right] \\
= & 2 \int_{\Sigma} d^{2} x[\Omega(\beta, \mathbf{x})-\Omega(0, \mathbf{x})] B(0, \mathbf{x}) .
\end{aligned}
$$

The magnetic $\left(B \equiv F_{12}\right)$ and electric fields, being physical, must be periodic in $t$. The electric contribution in Eq. (2.16) vanishes if $\Sigma^{2}$ does not allow nontrivial boundary conditions (the gauge invariant $E_{i}$ cannot have jumps, while $\Omega$ must also be a well-defined two-scalar on $\Sigma^{2}$ ). On the other hand, the magnetic term does not vanish for large transformations, where Eq. (2.16) becomes

$$
\Delta I_{\mathrm{CS}}=4 \pi n \int_{\Sigma^{2}} d^{2} x B(0, \mathbf{x})=4 \pi n \Phi_{\Sigma^{2}}(B) .
$$

The magnetic flux $\Phi$ is in general nonvanishing, time independent (by the Bianchi identity), and as a topological necessity [8], $\Phi / 2 \pi$ is integer quantized. It would thus seem that any bare CS action, conveniently defined as $\mu / 16 \pi^{2} I_{\mathrm{CS}}$, shifts by $\mu k n / 2$ under large gauge changes. Consequently, the requirement that the phase exponential of any action (the relevant object at the quantum level) be gauge invariant 
would seem to enforce the quantization condition ${ }^{2}$ on the CS parameter $\mu / 2 \pi$ that it be an even integer. Unfortunately, while this quantization argument is attractive, $I_{\mathrm{CS}}$ is not even well defined, precisely due to the very reason $\Phi \neq 0$ for quantization. Briefly stated, $\Phi \neq 0$ requires nontrivial connections $\mathbf{A}$ on $\Sigma^{2}$, thereby making $I_{\mathrm{CS}}$ manifestly patch dependent (a well-defined action is not patch-dependent). This major deficiency in $I_{\mathrm{CS}}$ should make one suspicious of the validity of the above quantization requirement. Fortunately, $I_{\mathrm{CS}}$ can be "improved," but we will see that the quantization of the (bare) coefficient of the improved $I_{\mathrm{CS}}$ becomes $\mu / 2 \pi$ $=n$ rather than $2 n$.

We now sketch a heuristic "derivation" of $I_{\mathrm{CS}}$, since precise ones were given long ago [9-11]; a new derivation [12] will also justify it. Consider the particular gauge transformation

$$
\Omega(t, \mathbf{x})=-\left[\int_{0}^{t} d t^{\prime}-\frac{t}{\beta} \int_{0}^{\beta} d t^{\prime}\right] A_{0}\left(t^{\prime}, \mathbf{x}\right) \equiv \mathcal{O} A_{0} \equiv \tilde{A}_{0}(t, \mathbf{x}),
$$

in its effect on $I_{\mathrm{CS}}$. Since $\Omega(t, \mathbf{x})$ is manifestly periodic in $t$ $[\Omega(\beta)=\Omega(0)=0]$, we are allowed to neglect the divergence in $\left(\Delta I_{\mathrm{CS}}\right)$ and thus $I_{\mathrm{CS}}(A)=I_{\mathrm{CS}}\left(A^{U}\right)$, where it is easy to check that the transformed fields are ${ }^{3}$

$$
\begin{aligned}
& A_{0}^{U}(t, \mathbf{x})=\frac{1}{\beta} \int_{0}^{\beta} d t^{\prime} A_{0}\left(t^{\prime}, \mathbf{x}\right)=\mathcal{A}_{0}(\mathbf{x}), \\
& A_{i}^{U}(t, \mathbf{x})=A_{i}(0, \mathbf{x})+\tilde{E}_{i}(t, \mathbf{x}) .
\end{aligned}
$$

In terms of these variables, $I_{\mathrm{CS}}$ has the form

$$
\begin{aligned}
I_{\mathrm{CS}}(A)= & 2 \int_{0}^{\beta} d t \int d^{2} x\left\{\mathcal{A}_{0}(\mathbf{x}) B(t, \mathbf{x})\right. \\
& \left.+\epsilon^{i j}\left[\widetilde{E}_{i}(t, \mathbf{x})+A_{i}(0, \mathbf{x})\right] E_{j}(t, \mathbf{x})\right\} \\
= & 2 \int_{0}^{\beta} d t \int d^{2} x\left[\mathcal{A}_{0}(\mathbf{x}) B(t, \mathbf{x})+\epsilon^{i j} \widetilde{E}_{i}(t, \mathbf{x}) E_{j}(t, \mathbf{x})\right. \\
& \left.+\epsilon^{i j} A_{i}(0, \mathbf{x}) \partial_{j} \mathcal{A}_{0}(\mathbf{x})\right] \\
= & 2 \int_{0}^{\beta} d t \int d^{2} x\left\{\mathcal{A}_{0}(\mathbf{x})[B(t, \mathbf{x})+B(0, \mathbf{x})]\right. \\
& \left.+\epsilon^{i j} \widetilde{E}_{i}(t, \mathbf{x}) E_{j}(t, \mathbf{x})\right\}-K, \\
K \equiv & -2 \int d^{3} x \epsilon^{i j} \partial_{j}\left[A_{i}(0, \mathbf{x}) \mathcal{A}_{0}(\mathbf{x})\right]
\end{aligned}
$$

where, to reach the last term of the second equality, we have used $E_{j}(t, \mathbf{x})=-\partial_{j} \mathcal{A}_{0}(\mathbf{x})+\partial_{0} A_{j}^{U}(t, \mathbf{x})$ and then dropped $\partial_{0} A_{j}^{U}(t, \mathbf{x})$ by periodicity. The sum $\bar{I}_{\mathrm{CS}} \equiv I_{\mathrm{CS}}+K$ is perfectly

\footnotetext{
${ }^{2}$ Mathematically this quantization relies on the fact that $\Pi_{1}[\mathrm{U}(1)]=\mathbb{Z}$. In the non-Abelian regime, quantization of $\mu / 2 \pi$ is of course always required [3].

${ }^{3}$ Note that the $\mathcal{O}$ operation projects out any time-independent factors.
}

well defined (and small gauge invariant) since it contains no explicit A dependence and represents the advertized "improved" CS term. The boundary term $K$ fails to vanish for interesting configurations, involving nontrivial flux $\Phi$, because there the connection $\mathbf{A}$ is different on the two patches that cover the sphere. ${ }^{4}$ [Note that perturbative calculations in the usual expansion about a trivial $\left(A_{\mu}=0\right)$ connection will never see the $K$ term; to include a reference background would complicate even the one-loop computation considerably.] We may now rewrite $I_{\mathrm{CS}}$ as the sum of terms depending only on the $F_{\mu \nu}$ together with those depending on the flat connection, from the constant part ${ }^{5}$ of $\mathcal{A}_{0}$ in Eq. (2.12),

$$
\bar{I}_{\mathrm{CS}}=8 \pi a \Phi+Q[F]=16 \pi^{2} n a+Q(F), \quad n \in \mathbb{Z} .
$$

This representation thus demonstrates that $\bar{I}_{\mathrm{CS}}$ is not independent, but is determined by $a$ and $F$, its behavior under large transformations being completely governed by $a$. It also enables us to compute the correct quantization of the coefficient in a bare $\bar{I}_{\mathrm{CS}}$ action (which must of course depend on $\bar{I}_{\mathrm{CS}}$, not $I_{\mathrm{CS}}$ ): Under a large gauge transformation, $a \rightarrow a$ $+1, \bar{I}_{\mathrm{CS}} \rightarrow \bar{I}_{\mathrm{CS}}+8 \pi \Phi$, that is, $\bar{I}_{\mathrm{CS}}$ changes by $16 \pi^{2} k$, so that the bare $\mu / 2 \pi$ must be an integer, not just an even one.

Having established the role of $\bar{I}_{\mathrm{CS}}$ as the carrier of the holonomy information, we return to the Fourier expansion of the action (2.14) and reexpress the $a$ dependence there in terms of $\bar{I}_{\mathrm{CS}}$ :

$$
\begin{aligned}
e^{-\Gamma\left(F, \bar{I}_{\mathrm{CS}}\right)}= & \sum_{k=-\infty}^{\infty}\left[\Gamma_{k}^{(1)}(F) \cos k\left(\bar{I}_{\mathrm{CS}} / 8 \pi n\right)\right. \\
& \left.+\Gamma_{k}^{(2)}(F) \sin k\left(\bar{I}_{\mathrm{CS}} / 8 \pi n\right)\right] .
\end{aligned}
$$

This form will be concretely realized by explicit field configurations in Sec. V. For our purposes, it shows how explicit CS terms can be present, when "protected" by sines and cosines, without loss of large invariance, but this invariance is lost in a power series expansion. As is necessary, we will confirm this formal analysis in Sec. III, when we obtain the properly regularized determinant.

We are now in a position to settle and old paradox arising in naive perturbative calculations of $\Gamma$ : At one-loop (which is everything if the photon is not dynamic) level, the fermions give rise to an effective CS contribution, irrespective of

\footnotetext{
${ }^{4}$ The patch dependence of $K$ is easily described schematically: Consider two patches defined by (for simplicity) some arbitrary latitude cut $\theta=\theta_{0}$. Then if $A^{ \pm}$denote the respective values of the $A_{\phi}\left(\theta_{0}\right)$ on the upper and lower caps, it is manifest that $K$ $\sim \int d \phi\left(A^{+}-A^{-}\right) \mathcal{A}_{0}\left(\theta_{0}\right)$. Clearly, $K$ depends on the patch choice $\theta_{0}$ and does not vanish if $\Phi \neq 0$, due to the usual nontrivial gauge gauge difference on the patches familiar from magnetic monopole constructions.

${ }^{5}$ Here another arbitrary choice was made in keeping the constant part of $\mathcal{A}$ even though it appeared in differentiated form in the second equality's last term. This choice of what physical term to divide between $K$ and $\bar{I}_{\mathrm{CS}}$ led to the coefficient shown in Eq. (2.22). Fortunately, this is also the correct answer from [9-12].
} 
whether there is an initial bare one. The calculation of the coefficient is straightforward [13],

$$
\frac{\Delta \mu}{2 \pi}=\frac{e^{2}}{2} \tanh \left[\frac{\beta m}{2}\right]
$$

which is noninteger for generic $\beta=(\kappa T)^{-1}$. However, since at the same time it was (correctly) thought both that Eq. (2.24) seems to signal an irremediable large gauge anomaly and that the matter action $I_{f}=i \int(d x) \bar{\psi}(\not D+m) \psi$ and the process of integrating out its excitations to obtain the effective action $\exp (-\Gamma[A])=\operatorname{det}(i \not D+i m)$ should be intrinsically gauge invariant, this paradox has generated a considerable literature. Opinions have differed widely: one claim is that there is no anomaly, due to some obscure nonperturbative mechanism that restores the integer nature of $\mu[14,15]$. Specifically, Ref. [14] conjectured that the usual perturbative definition of the CS coefficient through the two-point function is not physically relevant and a possible nonperturbative one in terms of the complete effective action was proposed. Given a large gauge transformation $U_{L}$ of winding number $n$, a new renormalized $\mu_{R}$ is to be defined according to $2 \pi n \mu_{R}=\Gamma\left[U_{L}^{-1} \partial_{\mu} U_{L}\right]-\Gamma[0]$, and its integer nature is supposed to be protected by some topological Ward identity. In Ref. [15], under the (incorrect) assumption that the only parity-violating contribution in the effective action is the CS term, it is shown that the path-integral formulation of the theory is consistent only if $\mu$ remains a temperatureindependent integer. Another point of view accepts the temperature dependence in Eq. (2.24) as a correct prediction of the theory entailing, for example, the breakdown of the anyonic description of superfluidity [4].

We have already seen how to dispel the paradox formally. A first step in understanding the real nature of this puzzle was recently taken in Ref. [16]; a solvable one-dimensional Abelian analogue of the problem was carefully analyzed and in particular its effective action was computed in closed form: While gauge invariant (at least for an even number of fermions), its perturbative expansion indeed contained a (one-dimensional) CS term with the temperature-dependent coefficient (2.24). This result thus allowed the coexistence of large gauge invariance of the full action and nonquantization of the perturbative CS coefficient. It was then established in Ref. [6], that the effective action, independent of the number of fermions, is indeed invariant under both small and large transformations using the classic results of Refs. $[17,18]$ that permit a clear definition of the Dirac operator's functional determinant by means of $\zeta$-function regularization, as we shall show in detail in Sec. III. We shall also see how ChernSimons term's noninvariance is precisely compensated by accompanying nonlocal contributions in the effective action that are not perturbatively visible.

Finally we mention another historical misunderstanding which goes back to the original papers, Refs. [19] and [20]: the relation between the number of fermions and gauge invariance in three dimensions. It is often stated that, in complete analogy with the $\mathrm{SU}(2)$ anomaly in four dimensions [21], large gauge invariance in three dimensions is maintained only for an even number of fermions or more precisely for a certain choice of matter multiplets $[16,22]$. What is true here is that in the even $N_{f}$ case one can define some- what different regularizations that preserve both gauge and parity, something that is indeed not achievable for odd $N_{f}$. With our regularization prescription, however, large gauge invariance is always preserved, while parity is always anomalous for both even and odd number of charges.

\section{THE ACTION: REGULARIZATION, REPRESENTATION, AND ANOMALIES}

We now turn to the implementation of the formal framework of Sec. II, by regularizing the fermion determinant and then exhibiting its properties. We shall review the definition of the Dirac operator's determinant in the rigorous framework of the $\zeta$-function approach $[17,18,23]$ for arbitrary dimension. Although this has become a very popular technique and a well-established mathematical subject, we believe it is worth reexamining in order to point out some subtleties peculiar to odd-dimensional manifolds. Specifically, we will stress the delicate interplay between spectral asymmetry, large gauge invariance, parity anomalies, and perturbative expansions. In the process a compact integral representation of the $\zeta$ function for massive electrons in terms of the massless gauge invariant heat kernels will be derived for all dimensions. It will enable us to provide, in Sec. IV, detailed expansion of both the parity odd and even parts for small and large fermion masses.

The mathematical tool that allows us to make sense of the formal product of the eigenvalues $\Pi_{\lambda_{n}} \lambda_{n}$ defining the determinant is $\zeta$-function regularization, which, for normal operators such as $i(\not D+m)$ on a compact manifold, reduces to

$$
\zeta(s) \equiv \sum_{\lambda_{n} \in \text { spectrum }}\left(\lambda_{n}\right)^{-s}
$$

in the sum each eigenvalue $\lambda_{n}$ in the spectrum is repeated according to its multiplicity. ${ }^{6}$ The convergence of the series (3.1) for $\operatorname{Re} s>d$ in $d$ dimensions is assured by a classical result on the asymptotic growth of eigenvalues [18], which for the massive Dirac operator reads

$$
\lim _{n \rightarrow \infty} n\left|\lambda_{n}\right|^{-d} \simeq \text { const. }
$$

Here the eigenvalue sequence is ordered so that $\left|\lambda_{0}\right| \leqslant\left|\lambda_{1}\right|$ $\leqslant \cdots$. Actually one can go further and show that $\zeta(s)$ for $s>d$ defines an analytic function that can be extended to a meromorphic function with only simple poles. In particular its analytic extension is regular at $s=0$ and its derivative

\footnotetext{
${ }^{6}$ There is an intrinsic ambiguity, the scale dependence of the dimensionful $\lambda_{n}$, hidden in Eq. (3.1). Strictly speaking, to construct the $\zeta$ function one should use the dimensionless ratio $\lambda_{n} / \mu$, with an arbitrary scale $\mu$. The determinant is therefore actually undetermined up to terms proportional to $\zeta(0) \ln \mu$ [23], namely, to the well-known trace anomaly. In odd dimensions this contribution of course vanishes. Note also that the extension to $N$ fermions simply involves the product of the individual determinants.
} 
there defines the determinant according to Hawking's relation $^{7}[23]$

$$
\operatorname{det} i(\not D+m)=\exp \left[-\zeta^{\prime}(0)\right] ; \quad \Gamma[A]=\zeta^{\prime}(0) .
$$

Since the complex power is a multivalued function, a careful definition of $\lambda_{n}^{-s}$ is required to avoid ambiguities in Eq. (3.1) and thence in Eq. (3.3). We take it to be $\exp \left(-s \ln \lambda_{n}\right)$ where the cut of the logarithm is chosen to be over the real positive axis, $0 \leqslant \arg \left(\lambda_{n}\right)<2 \pi$, enabling us to rewrite $\zeta(s)$ in the more convenient form

$$
\zeta(s)=\sum_{\operatorname{Re} \lambda_{n}>0}\left(\lambda_{n}\right)^{-s}+\exp (-i \pi s) \sum_{\operatorname{Re} \lambda_{n}<0}\left(-\lambda_{n}\right)^{-s}
$$

A different cut may alter the determinant [i.e., produce terms that are not proportional to its intrinsic ambiguity $\zeta(0)]$ only if it intersects the line $\operatorname{Im} z=m$ and thereby has crossed an infinite number of eigenvalues. In that case, instead of Eq. (3.4), one would have

$$
\zeta(s)=\sum_{\operatorname{Re} \lambda_{n}>0}\left(\lambda_{n}\right)^{-s}+\exp (i \pi s) \sum_{\operatorname{Re} \lambda_{n}<0}\left(-\lambda_{n}\right)^{-s} .
$$

Equation (3.5) has been rewritten by using the same cut as in Eq. (3.4) in order to compare them; we have also dropped contributions proportional to $\zeta(0)$. This alternative choice does not affect gauge invariance, but, as we shall see later, does change the sign of the possible parity anomaly terms in $\Gamma[A]$ as was noted in Ref. [24] by more complicated considerations. It represents the nonperturbative analogue of the more familiar sign ambiguity encountered in defining the perturbative series via, e.g., Pauli-Villars regularization. There, it appears as an explicit dependence on the sign $M /|M|$ of the regulator mass. ${ }^{8}$ We will return to the significance and fixing of the ambiguity.

Turning now to gauge invariance in this framework, it is clear that it hinges on that of the eigenvalue spectrum. But small transformations do not affect the $\lambda_{n}$ at all, while the large ones merely permute them, as in usual illustrations of index theorems [7]. Thus every well-defined symmetric function of the spectrum, such as $\zeta(s)$ and hence $\Gamma[A]$ is unchanged and so gauge invariant. This argument does not rely on the particular topology of the manifold we are considering, and it will hold for finite temperature space-times that are products of $S^{1}$ times a $(d-1)$-dimensional compact manifold.

\footnotetext{
${ }^{7}$ Although our discussion is focused on the Dirac operator, all the results extend, with slight modifications, to the larger class of the elliptic pseudodifferential operators [17] with a ray of minimal growth (Agmon ray).

${ }^{8}$ It has been pointed out that a larger ambiguity in the perturbative approach can be obtained by using more than one Pauli-Villars field [25]. This unnatural result has a (likewise unnatural) counterpart in $\zeta$-function regularization: use the well-known "product anomaly" $\operatorname{det}(A B) \neq \operatorname{det}(A) \operatorname{det}(B)$ to bring in definitions that differ in the number of determinants, each of whose cuts is to be separately fixed.
}

To investigate the properties of the determinant more closely, we must rewrite the "abstract" $\zeta$ function in terms of the well-established machinery of the heat-kernel equation. (This task is not completely trivial because our operator is not positive definite.) Let us illustrate this first in the massless case and then proceed to the massive one. This will also allow a simpler connection to earlier results.

At $m=0$, the eigenvalues being real, a parity transformation is simply

$$
\lambda_{n} \rightarrow-\lambda_{n}
$$

so we can decompose $\zeta(s)$ into

$$
\zeta(s) \equiv \zeta_{\mathrm{PC}}(s)+\zeta_{\mathrm{PV}}(s)
$$

parity even and odd parts,

$$
\begin{aligned}
\zeta_{\mathrm{PC}}(s) \equiv & \frac{1+\exp (\mp i \pi s)}{2} \sum_{\lambda_{n} \in \text { spectrum }}\left(\left|\lambda_{n}\right|\right)^{-s}, \\
\zeta_{\mathrm{PV}}(s) \equiv & \frac{1-\exp (\mp i \pi s)}{2} \\
& \times\left(\sum_{\lambda_{n}>0}\left(\lambda_{n}\right)^{-s}-\sum_{\lambda_{n}<0}\left(-\lambda_{n}\right)^{-s}\right),
\end{aligned}
$$

while the $\mp$ keeps track of the relevant ambiguity in changing cut. These two objects can be now easily related to the square $\mathbb{D}^{2}$ of the Dirac operator (the Laplacian on the spinors) and to the $\eta$ function of $I D$. Explicitly we have

$$
\begin{aligned}
& \zeta_{\mathrm{PC}}(s)=\frac{1+\exp (\mp i \pi s)}{2} \zeta_{D^{2}}(s / 2) \\
& \zeta_{\mathrm{PV}}(s)=\frac{1-\exp (\mp i \pi s)}{2} \eta(s)
\end{aligned}
$$

Both $\zeta_{D^{2}}(s)$ and $\eta(s)$ are well-defined and gauge-invariant quantities, which admit an explicit heat-kernel representation

$$
\begin{aligned}
\zeta_{D^{2}}(s)= & \frac{1}{\Gamma(s)} \int_{0}^{\infty} d t t^{s-1} \operatorname{Tr}\left[\exp \left(-t \mathbb{D}^{2}\right)\right] \\
\eta(s)= & \frac{1}{\Gamma[(s+1) / 2]} \int_{0}^{\infty} d t t^{(s+1) / 2-1} \\
& \times \operatorname{Tr}\left[\not D \exp \left(-t \mathbb{D}^{2}\right)\right]
\end{aligned}
$$

Since both functions are analytic ${ }^{9}$ at $s=0$, the Dirac determinant takes the form

$$
\operatorname{det}(\not D)=\exp \left[-\frac{1}{2} \zeta_{D^{2}}^{\prime}(0) \mp \frac{i \pi}{2} \eta(0) \pm \frac{i \pi}{2} \zeta_{D^{2}}(0)\right] .
$$

While the $\mp$ in front of $\eta(0)$ represents a relevant ambiguity in the definition of the determinant, the $\zeta_{D^{2}}(0)$ contribution

\footnotetext{
${ }^{9}$ While the regularity of $\zeta_{D}^{2}$ is to be expected, that of $\eta(0)$ is a nontrivial result and we refer the interested reader to Ref. [18].
} 
can be reabsorbed in the first term by choosing the scale parameter ${ }^{5}$ to be $\mu=-1$. [Note, in fact, that Eq. (3.10) implies $\zeta(0)=\zeta_{D^{2}}(0)$; also $\zeta(0)$ vanishes at odd $d$, being essentially the conformal anomaly.] In even dimensions the existence of $\gamma_{d+1}$, which anticommutes with the Dirac operator, entails the absence of spectral asymmetry and thus the vanishing of $\eta(0)$ so that also the first $\mp$ is harmless. In odd dimensions (no $\gamma_{d+1}$ ) no symmetry prevents us from having $\eta(0) \neq 0$ and consequently from having anomalous parityviolating terms in the effective action whose overall sign is not determined. Unlike $\zeta_{D^{2}}(0), \eta(0)$ cannot be reabsorbed as its parity is opposite to that of $\zeta_{D^{2}}^{\prime}(0)$. While $\eta(0)$ is a gauge-invariant functional of the field, it is neither local nor continuous. It can be explicitly computed with the help of the Atiyah-Patodi-Singer theorem (see, e.g., Ref. [7]) and consists of two parts: a continuous local functional given by the appropriate dimensional (improved) CS action plus a highly nonlocal discontinuous contribution given by the "index" $\pi\left(N_{+}-N_{-}\right)$. Here $N_{+}$is the number of positive eigenvalues that become negative as $A_{\mu}$ is continuously deformed to some reference (background) connection ${ }^{10} B_{\mu}$, and vice versa for $N_{-}$. Note that (large) gauge invariance is maintained through a cancellation between the CS action and the nonlocal index contribution as advertized earlier. The CS Lagrangian is a local polynomial of dimension $d$ in the fields and their derivatives, so it should, in principle, be removable, unlike the index. If we make this choice, we obviously lose large gauge invariance: under transformations of winding number $n$ the determinant is multiplied by a phase factor $\exp (i \pi n)$. Instead, parity invariance is recovered in spite of the surviving index contribution, because while the index changes sign under parity, it is of the form $i \pi \times$ an integer, which leaves the determinant unchanged. (The effective action actually changes by the acceptable phase $2 \pi i n$.)

We are now ready to deal with the massive case. Let us first note that the above massless parity decomposition still holds formally, but it has lost its physical meaning because a parity transformation here means

$$
\lambda_{n} \rightarrow-\lambda_{n}^{*}
$$

The eigenvalues have, in fact, become complex since the euclidean Dirac mass is anti-Hermitian: they are given by $\lambda_{n}+i m$, where $\lambda_{n}$ are those of the massless operator (and $i \not D$ is Hermitian). In this case, by means of the Mellin representation of the complex power, we can write, for the parity even and odd parts,

\footnotetext{
${ }^{10}$ While $B_{\mu}$ can be taken to be zero for trivial bundles, the interesting Abelian case as we have seen always involves a flux and hence nontrivial ones. In this context see, e.g., Ref. [7]. Indeed, as shown in Ref. [1] the introduction of the reference connection is another way to reach the correct $\bar{I}_{\mathrm{CS}}$.
}

$$
\begin{aligned}
\zeta_{\mathrm{PC}}(s)= & \frac{\exp \left(\mp i \frac{\pi s}{2}\right)}{\Gamma(s)} \int_{0}^{\infty} d t t^{s-1} \cos \left(m t \mp \frac{\pi s}{2}\right) \\
& \times \sum_{\lambda_{n}} \exp \left(-\left|\lambda_{n}\right| t\right), \\
\zeta_{\mathrm{PV}}(s)= & \frac{\exp [\mp i(\pi s / 2)]}{i \Gamma(s)} \int_{0}^{\infty} d t t^{s-1} \sin \left(m t \mp \frac{\pi s}{2}\right) \\
& \times\left[\sum_{\lambda_{n}>0} \exp \left(-\left|\lambda_{n}\right| t\right)-\sum_{\lambda_{n}<0} \exp \left(-\left|\lambda_{n}\right| t\right)\right] .
\end{aligned}
$$

The kernels in Eqs. (3.15) and (3.16) can be again written in term of the heat kernels of the square of the massless Dirac operator and of its $\eta$ function. We shall begin by considering the parity-conserving part $\zeta_{\mathrm{PC}}(s)$. The first step is to find a function $F(s, t)$ such that

$$
\begin{gathered}
\int_{0}^{\infty} d t t^{s-1} \cos \left(m t \mp \frac{\pi s}{2}\right) \exp (-\lambda t) \\
=\int_{0}^{\infty} d t F(s, t) \exp \left(-\lambda^{2} t\right) .
\end{gathered}
$$

This integral equation can be easily solved by interpreting it as an identity between Laplace transforms. In fact one can immediately write

$$
\begin{aligned}
F(s, t)= & \frac{1}{2 \pi i} \int_{\gamma-i \infty}^{\gamma+i \infty} d \lambda \exp (\lambda t) \int_{0}^{\infty} d p p^{s-1} \\
& \times \cos \left(m p \mp \frac{\pi s}{2}\right) \exp (-\sqrt{\lambda} p),
\end{aligned}
$$

where $\gamma$ is a real constant that exceeds the real part of all the singularities of the second integral. With the help of $F(s, t)$, the $\zeta_{\mathrm{PC}}(s)$ takes the form

$$
\begin{aligned}
\zeta_{\mathrm{PC}}(2 s) & =\frac{1}{\Gamma(s)} \int_{0}^{\infty} d t t^{s-1} K_{\mp}(t, s) \sum_{\lambda_{n}} \exp \left(-t \lambda_{n}^{2}\right) \\
& =\frac{1}{\Gamma(s)} \int_{0}^{\infty} d t t^{s-1} K_{\mp}(t, s) \operatorname{Tr}\left[\exp \left(-t \not D^{2}\right)\right]
\end{aligned}
$$

where

$$
\begin{aligned}
K_{\mp}(t, s) \equiv & \exp (\mp i \pi s)\left[K^{(1)}(t, s) \pm 2 m \sqrt{t} K^{(2)}(t, s)\right] \\
= & \exp (\mp i \pi s)\left[\cos (\pi s) \Phi\left(\frac{1}{2}+s, \frac{1}{2} ;-m^{2} t\right)\right. \\
& \pm 2 m \sqrt{t} \frac{\Gamma(1+s)}{\Gamma(s+1 / 2)} \\
& \left.\times \sin (\pi s) \Phi\left(1+s, \frac{3}{2} ;-m^{2} t\right)\right]
\end{aligned}
$$


and $\Phi(\alpha, \beta ; z)$ denotes the confluent hypergeometric function. Let us now perform the analogous analysis for the parity-violating contribution $\zeta_{\mathrm{PV}}(s)$. This time we need a function $F(s, t)$ satisfying the integral identity

$$
\int_{0}^{\infty} t^{s-1} \sin \left(m t \mp \frac{\pi s}{2}\right) \exp (-\lambda t)=\int_{0}^{\infty} F(s, t) \lambda \exp \left(-\lambda^{2} t\right) .
$$

The explicit form of this $F(s, t)$ can be constructed, as before, by means of the Laplace transform. In particular we get

$$
\begin{aligned}
F(s, t)= & \frac{1}{2 \pi i} \int_{\gamma-i \infty}^{\gamma+i \infty} \frac{d \lambda}{\sqrt{\lambda}} \exp (\lambda t) \int_{0}^{\infty} d p p^{s-1} \\
& \times \sin \left(m p \mp \frac{\pi s}{2}\right) \exp (-\sqrt{\lambda} p),
\end{aligned}
$$

with $\gamma$ is in Eq. (3.18). In terms of this new kernel, the parity-violating part becomes

$$
\begin{aligned}
\zeta_{\mathrm{PV}}(s)= & \pm \frac{i}{\Gamma[(s+1) / 2]} \int_{0}^{\infty} d t t^{(s-1) / 2} G_{\mp}(t, s) \\
& \times \sum_{\lambda_{n}} \lambda_{n} \exp \left(-t \lambda_{n}^{2}\right) \\
= & \pm \frac{i}{\Gamma[(s+1) / 2]} \int_{0}^{\infty} d t t^{(s-1) / 2} G_{\mp}(t, s) \\
& \times \operatorname{Tr}\left[\not D \exp \left(-t \not D^{2}\right)\right],
\end{aligned}
$$

with

$$
\begin{aligned}
G_{\mp}(t, s) \equiv & \exp \left(\mp i \frac{\pi s}{2}\right) \sin \left(\frac{\pi s}{2}\right) \\
& \times\left[G^{(1)}(t, s) \mp 2 m \sqrt{t} G^{(2)}(t, s)\right]
\end{aligned}
$$

$$
\begin{aligned}
& =\exp \left(\mp i \frac{\pi s}{2}\right) \sin \left(\frac{\pi s}{2}\right)\left(\Phi\left(\frac{s}{2}, \frac{1}{2} ;-m^{2} t\right)\right. \\
& \left.\quad \mp 2 m \sqrt{t} \frac{\Gamma(1-s / 2)}{\Gamma[(1-s) / 2]} \Phi\left(\frac{1+s}{2}, \frac{3}{2} ;-m^{2} t\right)\right) .
\end{aligned}
$$

Equations (3.19) and (3.23) are the promised "spectral" representations for the $\zeta$ and $\eta$ functions, and in particular the weights $G_{\mp}$ and $K_{\mp}$ encode all the information about the mass dependence of our determinant. [Actually they contain more, because they hold for all $s$ and not only at $s=0$.] Therefore they can be used to investigate the properties of the effective action in different mass limits. In the next section we shall use them to derive expansions of the effective action for small and large masses. With their help, one can also show that the general considerations developed in the massless case extend unchanged to the massive one.

\section{LARGE AND SMALL MASS EXPANSIONS} by

The parity-conserving part of the effective action is given

$$
\begin{aligned}
\Gamma_{\mathrm{PC}}[A]= & \left.\frac{d}{d s} \zeta_{\mathrm{PC}}(s)\right|_{s=0}=\left.\frac{1}{2} \frac{d}{d s} \zeta_{\mathrm{PC}}(2 s)\right|_{s=0}=\frac{1}{2} \frac{d}{d s} \\
& \times\left[\frac{1}{\Gamma(s)} \int_{0}^{\infty} d t t^{s-1} K_{\mp}(t, 0) \operatorname{Tr}\left[\exp \left(-t D^{2}\right)\right]\right]_{s=0} \\
& +\lim _{s \rightarrow 0} \frac{1}{\Gamma(s)} \int_{0}^{\infty} d t t^{s-1} \frac{d K_{\mp}}{d s}(t, s) \\
& \times \operatorname{Tr}\left[\exp \left(-t \not D^{2}\right)\right] .
\end{aligned}
$$

The limits $s \rightarrow 0$ in Eq. (4.1) is a delicate point, detailed in Appendix A. The final result is

$$
\Gamma_{\mathrm{PC}}[A]=\left.\frac{1}{2} \frac{d}{d s} \zeta_{D^{2}+m^{2}}(s)\right|_{s=0} \mp \frac{\pi i}{2} \zeta_{D^{2}+m^{2}}(0)+\left\{\begin{array}{l}
\Gamma_{\mathrm{loc}}^{\mathrm{odd}}[A]= \pm \operatorname{sgn}(m) \sqrt{\pi} \sum_{k=0}^{(d-1) / 2} \frac{(-2)^{k}}{(2 k+1) ! !}\left(m^{2}\right)^{k+1 / 2} H_{d-1-2 k}, \\
\Gamma_{\mathrm{loc}}^{\mathrm{even}}[A]=-\sum_{k=1}^{d / 2}\left[\sum_{j=1}^{k} \frac{(-2)^{j-1}}{j} \frac{1}{(2 j-1) ! !(k-j) !}\right](i m)^{2 k} H_{d-2 k},
\end{array}\right.
$$

where $H_{n}$ are the Seeley-de Witt [1] coefficients for the massless Laplacian on the spinor: $\operatorname{Tr}\left[\exp \left(-t \mathbb{D}^{2}\right)\right]$ $=\sum_{n=0}^{\infty} H_{n} t^{(n-d) / 2}$. That the nonlocal part of the parityconserving action $\left[1 / 2 \zeta_{D^{2}+m^{2}}^{\prime}(0)\right]$ is governed by the massive Laplacian might be expected, but, surprisingly, we have extra dimension-dependent local contributions coming from the $s$ derivative of the kernel $K_{ \pm}(s, t)$. Note that in odd dimensions, in contrast to the even ones, their sign depends on the choice of cut. This phenomenon will become more relevant for the parity-violating part.
The analysis of the parity-violating effective action is substantially easier due to the absence of singular contributions as $s \rightarrow 0$; one obtains

$$
\begin{aligned}
\Gamma_{\mathrm{PV}}[A]= & \left.\frac{d}{d s} \zeta_{\mathrm{PV}}(s)\right|_{s=0} \\
= & \lim _{s \rightarrow 0} \pm \frac{i}{\Gamma[(s+1) / 2]} \int_{0}^{\infty} d t t^{(s-1) / 2} \frac{d G_{\mp}(t, s)}{d s} \\
& \times \operatorname{Tr}\left[\not D \exp \left(-t \not D D^{2}\right)\right] .
\end{aligned}
$$


The derivative of the kernel $G_{\mp}(t, s)$ at $s=0$ can be explicitly computed and gives

$$
\begin{aligned}
\left.\frac{d G_{\mp}(t, s)}{d s}\right|_{s=0}= & \frac{\pi}{2}\left[\Phi\left(0, \frac{1}{2},-m^{2} t\right)\right. \\
& \left.\mp \frac{2}{\sqrt{\pi}} m \sqrt{t} \Phi\left(\frac{1}{2}, \frac{3}{2},-m^{2} t\right)\right] \\
= & \frac{\pi}{2}\left(1 \mp \frac{2 \operatorname{sgn}(m)}{\sqrt{\pi}} \int_{0}^{m \sqrt{t}} \exp \left(-z^{2}\right) d z\right) .
\end{aligned}
$$

Thus the parity-violating part of the action turns out to be (in odd $d$, where it exists)

$$
\begin{aligned}
\Gamma_{\mathrm{PV}}[A]= & \pm \frac{i \pi}{2} \eta(0)-i \operatorname{sgn}(m) \int_{0}^{\infty} \frac{d t}{\sqrt{t}} \\
& \times \operatorname{Tr}\left[\not D \exp \left(-t \mathbb{D}^{2}\right)\right] \int_{0}^{|m| \sqrt{t}} d z \exp \left(-z^{2}\right) \\
= & {[ \pm 1-\operatorname{sgn}(m)] \frac{i \pi}{2} \eta(0)+i \operatorname{sgn}(m) \int_{0}^{\infty} \frac{d t}{\sqrt{t}} } \\
& \times \operatorname{Tr}\left[\not D \exp \left(-t \mathbb{D}^{2}\right)\right] \int_{|m| \sqrt{t}}^{\infty} d z \exp \left(-z^{2}\right) .
\end{aligned}
$$

In $d=3$ a similar representation for $\Gamma_{\mathrm{PV}}[A]$ was given in Ref. [26]. There, the cut giving the plus sign was implicitly chosen. As we shall see below, this corresponds to requiring "decoupling," i.e., vanishing of $\Gamma$, as the fermion mass goes to $+\infty$. Note, again, that the sign in front of the parity anomaly is entirely dependent on the choice of branch. As is clear from its representation our $\Gamma_{\mathrm{PV}}$ differ from the oddmass part $\widetilde{\Gamma}_{\mathrm{PV}}$ of $\Gamma$,

$$
\widetilde{\Gamma}_{\mathrm{PV}}[A] \equiv \frac{1}{2}(\Gamma[A, m]-\Gamma[A,-m]),
$$

as $\Gamma_{\mathrm{PV}}$ has even mass parts as well (and $\Gamma_{\mathrm{PC}}$ odd ones). This $\widetilde{\Gamma}_{\mathrm{PV}}$ clearly cannot detect the intrinsic anomaly (the one at $m \equiv 0)$; as a result the possibility of decoupling in the infinite mass limit is not manifest. (Also in a nonflat background geometry or higher dimensions, the above definition actually contains parity-conserving terms.)

Both Eqs. (4.2) and (4.5) can be used as starting points for a mass expansion of the theory. Let us first consider the small mass limit: in the parity-conserving case we simply have to Taylor-expand $\zeta_{D^{2}+m^{2}}(s)$ in power of mass. For odd dimension

$$
\Gamma_{\mathrm{PC}}^{\mathrm{odd}}[A]=\frac{1}{2} \frac{d}{d s} \zeta_{D^{2}}(0)+\sum_{k=1}^{\infty}(i m)^{2 k} \frac{\zeta_{D^{2}}(k)}{2 k}+\Gamma_{\mathrm{loc}}^{\mathrm{odd}}[A]
$$

where $\Gamma_{\text {loc }}^{\text {odd }}[A]$ is specified in Eq. (4.2). The appearance of the even power can be understood as a consequence of the behavior of the Dirac mass term under parity. Instead, the local contributions ( $\Gamma_{\operatorname{loc}}^{\mathrm{odd}}[A]$ ), proportional to $\mathrm{m}^{2 k+1}$, originate from a compensation between vanishing and divergent terms as $s$ goes to zero. The even dimensional case is more delicate, due to the fact that $\zeta_{D^{2}}(s)$ has in general simple poles for $n=1,2, \ldots, d / 2$. The final result can be presented in the form

$$
\begin{aligned}
\Gamma_{\mathrm{PC}}[A]= & \frac{1}{2} \frac{d}{d s} \zeta_{D^{2}}(0)+\sum_{k=d / 2+1}^{\infty}(i m)^{2 k} \frac{\zeta_{D^{2}}(k)}{2 k} \\
& +\sum_{k=1}^{d / 2} \frac{1}{2} \frac{d}{d s}\left[\frac{1}{\Gamma(s)} \int_{0}^{\infty} d t t^{s+n-1} \operatorname{Tr}\left(\exp -t \mathbb{D}^{2}\right)\right] \\
& +\Gamma_{\operatorname{loc}}^{\mathrm{even}}[A] .
\end{aligned}
$$

Analogously, Taylor-expanding the parity-violating part, we obtain

$$
\Gamma_{\mathrm{PV}}[A]= \pm i \frac{\pi}{2} \eta(0)-i \sum_{k=0}^{\infty}(-1)^{k} \frac{m^{2 k+1}}{2 k+1} \eta(2 k+1) .
$$

Note the presence of the intrinsic parity anomaly term $\pm i(\pi / 2) \eta(0)$ : it is the only one proportional to an even, $m^{0}$, power of the mass. We have already stated that it contains the CS action, but this does not mean that there are no other CS contributions hidden in the rest of the series. The large mass analysis below and the examples in Sec. $\mathrm{V}$ will indicate that they are actually present. Furthermore their coefficients are obviously mass and consequently temperature dependent (the mass can appear only through a dimensionless combination such as $\beta m$, though other combination are possible if there are other relevant scales in the problem, e.g., the volume of the manifold). On the other hand, gauge invariance is entirely unaffected by this: each term in the series is manifestly gauge invariant, since $\eta(s)$ is. The large mass limit is a more delicate issue, corresponding to an asymptotic expansion of the action. In the case of $\Gamma_{\mathrm{PC}}$, a simple application of Watson's lemma ${ }^{11}$ gives in the odd-dimensional case

$$
\begin{aligned}
\Gamma_{\mathrm{PC}}[A]= & \sqrt{\pi}[-1 \pm \operatorname{sgn}(m)] \\
& \times \sum_{n=0}^{(d-1) / 2} \frac{(-2)^{n}}{(2 n+1) ! !}\left(m^{2}\right)^{n+1 / 2} H_{d-1-2 n} \\
& +\frac{1}{2} \sum_{n=(d+1) / 2}^{\infty} \frac{\Gamma\left(n-\frac{d}{2}\right)}{\left(m^{2}\right)^{n-d / 2}} H_{2 n},
\end{aligned}
$$

while in even dimensions we have

\footnotetext{
${ }^{11}$ It essentially states [27] that an asymptotic expansion in $m$ of integrals such as $\int_{-\infty}^{\infty} d t e^{-t m^{2}} f(t)$ can be obtained by integrating the asymptotic expansion of $f(t)$ term by term.
} 


$$
\begin{aligned}
\Gamma_{\mathrm{PC}}[A]= & \sum_{n=}^{\infty} \frac{\Gamma(n-d / 2)}{\left(m^{2}\right)^{n-d / 2}} H_{2 n} \\
& +\frac{1}{2} \sum_{n=0}^{d / 2-1} \frac{\left(-m^{2}\right)^{d / 2-n}}{\Gamma(d / 2+1-n)} \\
& \times\left[\sum_{k=1}^{d / 2-n} \frac{1}{k}-\ln \frac{m^{2}}{\mu^{2}}\right] H_{2 n}-\frac{1}{2} \ln \frac{m^{2}}{\mu^{2}} H_{d} \\
& -\sum_{n=0}^{d / 2-1}\left(-m^{2}\right)^{d / 2-n} \\
& \times\left[\sum_{k=1}^{d / 2-n} \frac{1}{(2 k-1) ! !} \frac{(-2)^{k-1}}{k(d / 2-n-1) !}\right] H_{2 n} .
\end{aligned}
$$

Essentially, to obtain Eqs. (4.10) and (4.11), one expands the kernel $\operatorname{Tr}\left[\exp \left(-t \mathbb{D}^{2}\right)\right]$ for small $t$ and integrates term by term. The asymptotic nature of this series means that terms exponentially small in the mass, i.e., $O\left(e^{-\beta m}\right)$, cannot be seen. This can have quite dramatic consequences, as we will show through explicit examples in Sec. V. Nevertheless the expansion is both large and small gauge invariant order by order. In Eqs. (4.10) and (4.11) we have inserted the explicit form of the local terms: we mention first that in odd dimensions the divergent contributions (in the large mass limit) are nonvanishing only when gravity (through the geometry of the manifold) is involved. This can be inferred from the structure of the heat-kernel coefficients: for example, in three dimensions $H_{1}$ and $H_{2}$ correspond to the cosmological term and to the Einstein action, respectively. In general their coefficient is strongly dependent on the cut. For positive mass, the branch chosen in Eq. (3.4) gives zero (i.e., the fermion decouples), while the one in Eq. (3.5) would give a limit value of 2 (no decoupling). For negative mass, the reverse situation occurs with coefficient $(-2,0)$. This shows vividly that the choice of the cut is not just a matter of convention, but affects physical predictions. It is interesting to notice that in $d=3$ the first nontrivial correction to the infinite mass limit (the $H_{4}$ coefficient) is a Maxwell $\left(F_{\mu \nu}^{2}\right)$ term, with coefficient $1 / 48 \pi|m|$, in agreement with earlier calculations $[19,26,28]$. In the even-dimensional case the expansion is independent of the cut, as one would expect, and also involves logarithmic dependence on the mass, due to the nonvanishing of the trace anomaly there.

The analysis of the behavior of the parity-violating part is more intricate, and a straightforward application of Watson's lemma is not possible. However, looking at Eq. (4.5), one realizes that, for large mass, only small $t$ can contribute. The large $t$ behavior is, in fact, suppressed by the vanishing of the error function. Thus we can again expand the kernel and integrate term by term. This time we use the heat-kernel expansion $\operatorname{Tr}\left[\not D \exp \left(-t \mathbb{D}^{2}\right)\right]=\sum_{n=0}^{\infty} P_{n} t^{(n-d-1) / 2}$, where $P_{n}$ are different from zero only for odd $n$. We therefore obtain

$$
\begin{aligned}
\Gamma_{\mathrm{PV}}(A)= & {[-1 \pm \operatorname{sgn}(m)] \frac{i \pi}{2} \eta(0) } \\
& +i \sum_{n=0}^{\infty} \frac{P_{2 n+1}}{\left(m^{2}\right)^{n-(d-1) / 2}} \frac{\Gamma(n-d / 2+1)}{2 n+1-d} .
\end{aligned}
$$

Let us stress again the asymptotic nature of this series. The (local and invariant functionals of the gauge fields and of the geometry) $P_{2 n+1}$ coefficients differ from zero only for $n$ $>(d-1) / 2$. In the limit of infinite mass, the only possible surviving term is therefore proportional to the gauge invariant $\eta(0)$, but different coefficients are possible, in complete analogy with the parity conserving sector: $(2,0)$ for large positive mass and the cut as in Eq. (3.4); $(0,-2)$ for large negative mass and the cut as in Eq. (3.5). Thus, given a sign of the fermion mass, the branch can be always chosen so that the fermion completely decouples (or not!) in the infinite mass limit. This double pair of possibilities completely mimics the analogous perturbative result in the presence of one Pauli-Villars regulator. There the final asymptotic result would have been

$$
\Gamma_{\mathrm{PV}}[A] \simeq[\operatorname{sgn}(m)+\operatorname{sgn}(M)] I_{\mathrm{CS}},
$$

where $m$ is the fermion mass, while $M$ is the mass of the regulator. For $m$ positive, we have $(2,0)$ as $M \rightarrow(+\infty$, $-\infty)$, for $m$ negative we have instead $(0,-2)$ as $M \rightarrow$ $(+\infty,-\infty)$. The absence of the index in the perturbative result implies the loss of manifest gauge invariance for finite masses since $I_{\mathrm{CS}}$ has no counterpart to restore it (nor does it acquire the required boundary terms needed to make it well defined).

\section{EXPLICIT GAUGE FIELD EXAMPLES}

For concrete illustrations of how the perturbative paradox is circumvented, let us now consider some explicit examples of actions and large gauge transformations. We start by reviewing, according to Ref. [6], the $(0+1)$-dimensional toy model of Ref. [16]. It consists of $N$ fermions on a circle of a radius $\beta$ interacting with a $\mathrm{U}(1)$ field through the Lagrangian

$$
\mathcal{L}=\sum_{i=1}^{N} \bar{\psi}_{i}(t)\left(i \frac{d}{d t}+A(t)+i m\right) \psi_{i}(t) .
$$

The large transformations are taken to be

$$
U(t)=\exp [i f(t)]
$$

where $f(\beta)-f(0)=2 \pi n$.

The integer $n$ is the winding number of the map $U(t)$, i.e., $2 \pi i n=\int_{0}^{\beta} d t U(t)^{-1} U^{\prime}(t)$. The analog of parity in three dimensions is here charge conjugation $A \rightarrow-A$; while massless fermions are invariant, massive ones violate this symmetry. [Had a bare CS term, here $k A(t)$, been present in Eq. (5.1), invariance of the path integral under large transformations would require that $k$ in Eq. (5.1) be quantized, entirely as in $D=3$.] 
The eigenvalue problem corresponding to the $(0+1)$-dimensional Dirac operator can be exactly solved:

$$
\lambda_{n}=\frac{2 \pi}{\beta}\left(n-\frac{1}{2}\right)+\frac{2 \pi}{\beta} a+i m, n \in \mathbb{Z},
$$

where $a$ is the average of $A: a=(1 / 2 \pi) \int_{0}^{\beta} A(t) d t$. The $\zeta$ function can be computed in closed form in terms of the Hurwitz function [29] $\zeta_{H}(s, q)$

$$
\begin{aligned}
\zeta(s)= & N\left(\frac{\beta}{2 \pi}\right)^{s}\left[\zeta_{H}\left(a+\frac{1}{2}+i \frac{\beta m}{2 \pi}, s\right)\right. \\
& \left.+\exp (\mp i \pi s) \zeta_{H}\left(\frac{1}{2}-a-i \frac{\beta m}{2 \pi}, s\right)\right] .
\end{aligned}
$$

Throughout, the $\mp$ keeps track of the relevant ambiguity in choice of cut. The determinant is now easily evaluated directly from its definition,

$$
\begin{aligned}
\exp [-\Gamma(A)]= & \operatorname{det}\left(i \frac{d}{d t}+A(t)+i m\right) \\
= & \exp \left[-\zeta^{\prime}(0)\right] \\
= & {\left[2 \left(\cosh \left(\frac{\beta m}{2}\right) \cos \pi a\right.\right.} \\
& \left.\left.-i \sinh \left(\frac{\beta m}{2}\right) \sin \pi a\right) \exp \left( \pm i \pi a \mp \frac{\beta m}{2}\right)\right]^{N} \\
\equiv & {\left[1+e^{ \pm(2 \pi i a-\beta m)}\right]^{N} . }
\end{aligned}
$$

Note that this action depends on $a$ only via the $S^{1}$ holonomy $\exp (2 \pi i a)$ and thus is manifestly gauge invariant under a large transformation, $a \rightarrow a+1$, for either cut and for any $N$, even or odd. In the middle term this occurs through a sign cancellation between the separate factors. Though the value of final expression in Eq. (5.5) seems to depend completely on the choice of cut, the intermediate equality makes it clear that only the charge conjugation anomalous contribution is affected, in agreement with the general results of Sec. III. Notice also the necessary presence of an "intrinsic', (i.e., even present at $m=0)$ charge conjugation anomaly $\operatorname{Im} \Gamma_{\mathrm{CV}}[A]=i N(a-[a])$, where $[a]$ denotes the integer part of $a$. This is what allows us to preserve large gauge invariance independently of $N$. This result also clearly exhibits what was claimed on general grounds in Sec. III for the parity anomalous contribution, namely, the $\eta(0)$ : only the combination of the continuous part, given by the CS action $a$ and the discontinuous contribution coming from the index [a] is gauge invariant. Had we opted instead (as in Ref. [16]) for the $(0+1)$ equivalent of the more usual $C$-preserving regularization, the $\exp (i N \pi a)$ factor in Eq. (5.5) would have been missing and only even $N$ would have kept invariance, just as in $(2+1)$.

Dimension $(0+1)$ is also a good laboratory for testing the mass expansions discussed in Sec. IV and in particular that for large mass. If we apply the one-dimensional analogue of parity conserving or violating expansions (4.10) and (4.12) [or directly from Eq. (5.5)], we obtain

$$
\Gamma[A] \simeq(0,-2)\left[i \pi(a-[a])-\frac{\beta m}{2}\right], m>0,
$$

$$
\Gamma[A] \simeq(2,0)\left[i \pi(a-[a])-\frac{\beta m}{2}\right], \quad m<0 .
$$

This is a concrete realization of what was stated at the end of Sec. IV. Let us also notice that all the $1 / \mathrm{m}$ corrections are identically zero. One can understand this result from two different points of view. First, beyond the terms shown in Eqs. (5.6) and (5.7), all the others are exponentially small in the mass and thus they cannot affect the asymptotic series. Secondly, the one-dimensional Dirac operator coupled to a gauge field is always locally gauge equivalent to the free one [since locally $\left.A(t)=\partial_{t} B(t)\right]$. This means that the local coefficient of its heat-kernel expansion must be trivial, and dramatically shows how much information can be lost in a large mass expansion, even though the final result is gauge invariant. In other words, when topological degrees of freedom such as $a$ are involved, an expansion in the local coefficients of the heat kernel can rarely retain the complete dynamics of the theory.

Though very instructive because of its soluble nature, one might wonder if the mechanism realized in the toy $(0+1)$ model is shared by its three-dimensional counterpart, where a complete solution of the theory is not at our disposal. A more realistic example in this direction is to consider a purely magnetic configuration with flux $\Phi(B)=2 \pi n$ in $d$ $=3$. It is an easy exercise to show that the most general potential, up to a gauge transformation, generating such a field is

$$
A_{\mu} \equiv\left(\frac{2 \pi}{\beta} a, \mathbf{A}(\mathbf{x})\right)
$$

where $a$ is a flat $S^{1}$ connection and the two-potential $\mathbf{A}$ is static, living on the two-dimensional Riemann manifold $\Sigma^{2}$. The large transformations are associated to the $S^{1}$ map $a$ $\rightarrow a+1$, as in the $(0+1)$-dimensional case. (For $\Sigma$ of genus greater than 0 , large transformations corresponding to the nontrivial cycles of $\Sigma$ can be also constructed, but we will not discuss them. Here we will only be interested in the ones relevant in the finite temperature regime.)

We now proceed to compute the partition function for a single Dirac fermion in the background (5.8). Since the latter is time-independent, we can decouple $t$ by looking for eigenvectors of the form

$$
\hat{\psi}(t, x, y)=\exp \left[-\frac{2 \pi}{\beta}\left(n+\frac{1}{2}\right) t\right] \psi(x, y) .
$$

(In finite temperature field theory the integer factor $n$ in the phase is usually known as the Matsubara frequency.) The $\frac{1}{2}$ factor takes care of the antiperiodic boundary conditions for the fermion. The eigenvalue problem for the $d=3$ operator $i$ ID thus reduces to an infinite series of effective twodimensional ones parametrized by $n$,

$$
i \not D \psi=i \not D \psi+\frac{2 \pi}{\beta}\left(\alpha_{0}+n+\frac{1}{2}\right) \gamma^{0} \psi=(\lambda-i m) \psi \equiv \hat{\lambda} \psi
$$


Here $i \hat{D}$ is the massless Dirac operator on the twodimensional manifold $\Sigma$. The key observation is that the spectrum of $\mathcal{D}$ can be reconstructed once that of $\hat{D}$ is known. In fact let

$$
\phi(x, y)=\left(\phi_{1}(x, y), \phi_{2}(x, y)\right)
$$

be a (two-component) eigenvector of $\hat{\mathbb{D}}$, with eigenvalue $\mu$ $\neq 0$. Then the vectors

$$
\begin{aligned}
\psi_{ \pm}(x, y)= & \left(\phi_{1}(x, y), C_{ \pm} \phi_{2}(x, y)\right), \\
C_{ \pm} \equiv & -\frac{2 \pi}{\beta \mu}\left(\alpha_{0}+n+\frac{1}{2}\right) \\
& \pm \sqrt{\frac{4 \pi^{2}}{\beta^{2} \mu^{2}}\left(a+n+\frac{1}{2}\right)^{2}+1}
\end{aligned}
$$

are eigenvectors of $\mathbb{D}$ with eigenvalues

$$
\lambda_{( \pm)}=i m \pm \sqrt{\frac{2 \pi}{\beta}\left(a+n+\frac{1}{2}\right)^{2}+\mu^{2}} .
$$

As might be expected from the $\gamma_{0}$ in Eq. (5.10), each nonvanishing eigenvalue of $\hat{D}$ generates two eigenvalues of $\mathcal{D}$ of equal multiplicity. This symmetrical behavior suggests that they will not produce a spectral asymmetry and thus play no role in the clash between invariances under large and parity transformation. In fact, by using the representation (3.16) for $\zeta_{\mathrm{PV}}(s)$, it is immediate to see that their contribution there vanishes.

We come now to $\operatorname{Ker} \hat{D}$. The Atiyah-Singer theorem tells us that it is spanned by $\nu_{+}$spinors $\phi_{+}^{0}(x, y)$ with positive chirality and $\nu_{-}$spinors $\phi_{-}^{0}(x, y)$ with negative chirality, where $\nu_{+}-\nu_{-}=n$ is the flux of the $\mathbf{A}$; chirality is defined by $\gamma^{0} \phi_{ \pm}^{0}(x, y)= \pm \phi_{ \pm}^{0}(x, y)$. Both $\phi_{+}^{0}(x, y)$ and $\phi_{-}^{0}(x, y)$ are eigenvectors of $\mathcal{D}$ as well, but with eigenvalues

$$
\lambda_{( \pm)}^{0}=i m \pm \frac{2 \pi}{\beta}\left(n+a+\frac{1}{2}\right) .
$$

The chiral asymmetry of the $\operatorname{Ker} \hat{D}$ is inherited by the spectrum of $\mathbb{D}$ : in fact $\lambda_{-}^{0}$ and $\lambda_{+}^{0}$ have different degeneracy. This, as we shall see, will give rise to a nonvanishing anomalous parity contribution.

The ensuing $\zeta$ function is ${ }^{12}$

\footnotetext{
${ }^{12}$ Having already noticed that no asymmetry is entailed by the eigenvalues $\lambda_{( \pm)}$, we have written $\Sigma\left(\lambda_{(+)} \lambda_{(-)}\right)^{-s}$ instead of $\Sigma\left(\lambda_{(+)}\right)^{-s}+\Sigma\left(\lambda_{(-)}\right)^{-s}$. In fact, in absence of spectral asymmetry, these two quantities coincide up to local terms. The difference, proportional to the volume of $\Sigma$ in this case, can be evaluated with the help of the spectral representation given in Sec. III.
}

$$
\begin{aligned}
\zeta(s)= & \nu_{+} \zeta_{H}\left(\frac{1}{2}+a+i \frac{\beta m}{2 \pi}, s\right) \\
& +\exp (-i \pi s) \nu_{+} \zeta_{H}\left(\frac{1}{2}-a-i \frac{\beta m}{2 \pi}, s\right) \\
& +\nu_{-} \zeta_{H}\left(\frac{1}{2}-a+i \frac{\beta m}{2 \pi}, s\right) \\
& +\exp (-i \pi s) \nu_{-} \zeta_{H}\left(\frac{1}{2}+a-i \frac{\beta m}{2 \pi}, s\right) \\
& +\sum_{n, \mu_{k}}\left[m^{2}+\frac{4 \pi^{2}}{\beta^{2}}\left(a+n+\frac{1}{2}\right)^{2}+\mu_{k}^{2}\right]^{-s}
\end{aligned}
$$

where the discrete sum runs over $n \in \mathbb{Z}$ and $\mu_{k} \in[\operatorname{Spec}(\hat{D})$ $-\operatorname{Ker}(\hat{D})]$. Let us denote the sum term by the symbol $\zeta \hat{D}^{2}(s)$ even though that identification is not entirely correct. The determinant can be then computed and we obtain ${ }^{13,14}$

$$
\begin{aligned}
\exp [-\Gamma(A)]= & {[\exp (-\beta m+2 \pi i a)+1]^{\nu_{+}} } \\
& \times[\exp (-\beta m-2 \pi i a)+1]^{\nu_{-}} \\
& \times \exp \left[-\zeta_{\hat{D}^{2}}^{\prime}(0)\right] .
\end{aligned}
$$

From Eq. (5.16) it is manifest that the determinant splits in the product of two $(0+1)$-dimensional contributions and a reduced expression depending on $\mathbf{A}, \Sigma$, and the flat connection $a$. Amusingly, one can go further and partially compute $-\zeta_{\hat{D}^{2}}^{\prime}(0)$, namely, perform the sum over $n$. To this end, one first defines a Mellin representation of the complex power and then Poisson resums the series in $n$ (see Appendix B). In this way, we end up with a series for $\zeta_{\hat{D}^{2}}(s)$ that is analytic at $s=0$ and whose derivative at $s=0$ leads to

$$
\begin{aligned}
& \exp \left(-\zeta_{\hat{D}^{2}}(s)\right)=\left|\prod_{\mu_{k}}\left[1+\exp \left(-\beta \sqrt{\mu_{k}^{2}+m^{2}}+2 \pi i a\right)\right]\right|^{2} \\
& \times \exp \left[2 \pi \mathcal{F}-\left(\nu_{+}+\nu_{-}\right) m \beta\right] \\
& \mathcal{F} \equiv \zeta_{\left(\beta^{2} / 4 \pi^{2}\right)\left(\hat{\mathbb{D}}^{2}+m^{2}\right)}(-1 / 2)
\end{aligned}
$$

That the above infinite product is convergent follows immediately from the estimate (3.2). We have thus provided the explicit general form (5.16),(5.17), for the complete effective action in the background (5.8). It is a trivial exercise to compute in particular its parity-violating part (under $a \rightarrow-a$ ). The term governed by $\zeta_{\hat{D}^{2}}(s)$ is unaffected, so we obtain

\footnotetext{
${ }^{13}$ After the derivation in Ref. [6] of the general form (5.16) for the effective action, its odd-mass part $\widetilde{\Gamma}_{\mathrm{PV}}$ (4.6) (rather than the true $\Gamma_{\mathrm{PV}}$ itself) was recalculated in Ref. [30a] in a different way. The result there, which was its main content, was incorrect. Upon private explanation of their mistake to the authors, a second, corrected, version [30b] properly acknowledged our corrections. However, that acknowledgment did not survive in the published version [30c], nor in its erratum [30d] stating the true date of the revised version [30c].

${ }^{14} \mathrm{~A}$ recent computation [31] of $\widetilde{\Gamma}_{\mathrm{PV}}$ agrees with that implied by Eq. (5.16).
} 


$$
\begin{aligned}
\Gamma_{\mathrm{PV}}[A]= & \left(\nu_{+}-\nu_{-}\right)\left\{\arctan \left[\tan \left(\frac{\beta m}{2}\right) \tan [\pi(a-[a])]\right]\right. \\
& \pm \pi(a-[a])\} .
\end{aligned}
$$

The above equation exhibits the remarkable property of $\Gamma_{\mathrm{PV}}[A]$ that it factorizes into a part dependent only on the holonomy $a$ times one that involves, through $\left(\nu_{+}-\nu_{-}\right)$, the flux $\Phi$ on $\Sigma^{2}$ since as we saw $\left(\nu_{+}-\nu_{-}\right)=\Phi / 2 \pi$. This is both in accord with our initial "Fourier" representation as well as a general consequence of the index theorem on product manifolds (for details see Ref. [18], p. 288). [We have written the redundant combination $(a-[a])$ rather than $a$ in the argument of the tan above to emphasize its fundamental role.]

A simple but interesting special case of Eq. (5.8) where the eigenvalues $\mu_{k}$ are known explicitly is the instanton on the flat unit torus: $A_{i}=-\pi n \epsilon_{i j} x^{j}$. Here $\mu_{k}^{2}=4 \pi|n k|$ with $2 n$ degeneracy, while $2 \pi \zeta_{\left(\beta^{2} / 4 \pi^{2}\right)\left(\hat{D}^{2}+m^{2}\right)}(-1 / 2)$ $=n(4 \pi n)^{1 / 2} \beta \zeta_{H}\left(-1 / 2, m^{2} / 2 \pi n\right)-\left(\nu_{+}+\nu_{-}\right) m \beta$. Substituting into Eq. (5.17) we obtain

$$
\begin{aligned}
\exp [- & \Gamma(A)] \\
= & \left|\prod_{k=1}^{\infty}\left(1+\exp \left(-\beta \sqrt{4 \pi|n k|+m^{2}}+2 \pi i a\right)\right)\right|^{4 n} \\
& \quad \times \exp \left[n(4 \pi n)^{1 / 2} \beta \zeta_{H}\left(-1 / 2, \frac{m^{2}}{2 \pi n}\right)-2 m n \beta\right] .
\end{aligned}
$$

There are a number of other informative general properties to be drawn from Eqs. (5.16),(5.17). First their invariance under $a \rightarrow a+1$ is manifest and its structure is consistent with Eq. (2.14). Second, it is clear that a perturbative (i.e., in powers of $a$ ) expansion of Eqs. (5.16) and (5.17) loses periodicity in $a$ and hence does not see large invariance order by order. For example the Chern-Simons term $\left(I_{\mathrm{CS}}=\pi a n\right)$ has a coefficient $1-\tanh (\beta m / 2)$. The usually quoted coefficient omits the 1 that represents the intrinsic parity-anomaly price of our gauge-invariant regularization and hence persists at $m=0$. As we saw in Sec. III there is actually an ambiguity in its sign (reflecting a choice of cut), also present in other regularizations, for example, through the factor $\lim _{M \rightarrow \pm \infty} \operatorname{sgn}(M)$ in Pauli-Villars, even at perturbative Feynman diagram level. As we discussed, with our intrinsic parity-violating gaugepreserving choice the ambiguity is physically reflected in the degree of decoupling of a heavy fermion.

\section{CONCLUSIONS}

After first deriving a generic form for the Abelian gauge field effective action in arbitrary dimensions purely on gauge invariance grounds, we were able to represent it in detail using $\zeta$-function regularization. In the process, we found a uniform preservation of gauge invariance, under both small and large transformations, which in odd dimensions is linked with parity anomalies due to the possibility of spectral asymmetry. We thereby connected the machinery of index theorems to the more prosaic question of how improved Chern-
Simons terms (that carry the large gauge information) could be present in the finite temperature thermal field theory regime without violating the overall gauge invariance; this was closely related to the $\eta$ function. From our original representations, we were able to give "spectral" representations for the massive Dirac determinant in terms of the massless $\zeta$ and $\eta$ functions. In turn, this enabled us to provide explicit expansions for both parity even and odd parts of the effective action in the small as well as large mass limit. A number of subtleties inherent in these expansions were discussed. One important aspect is that there is a finite regularization ambiguity in the full nonperturbative action that parallels the well-known perturbative one where there is a residual PauliVillars regulator ambiguity: results depend on the sign of its mass even as it tends to infinity. For us, the ambiguity was in a twofold possibility of complex plane cut. A physically appealing choice was to insist on "decoupling" as the electron's mass becomes infinite. These ambiguities differ from the usual polynomial freedom associated with regularization, simply because there are no gauge-invariant polynomials available here. Instead, they are reflected in the nearest possible way to that: through the discrete value of the coefficient of $\eta(0)$, which contains the local, "polynomial" CS term. In connection with the importance of the flat direction as representing the large gauge aspects, we noted that these aspects would only be found in perturbative diagrams if one used fermion propagators in the "flat potential vacuum" rather than simply the usual free ones. Finally, we provided some explicit gauge field configuration examples to show the emergence of our general results in concrete cases involving external fields.

\section{ACKNOWLEDGMENTS}

This work is supported by NSF Grant No. PHY-9315811, in part by funds provided by the U.S. DOE under Cooperative Agreement No. DE-FC02-94ER40818 and by INFN, Frascati, Italy.

\section{APPENDIX A}

We start by considering $\Gamma_{\mathrm{PC}}[A]$ defined in Eq. (4.1). Recalling that

$$
K_{\mp}(t, 0)=\Phi\left(\frac{1}{2}, \frac{1}{2},-m^{2} t\right)=\exp \left(-m^{2} t\right),
$$

the first term in Eq. (4.1) can be cast as

$$
\left.\frac{1}{2} \frac{d}{d s} \zeta_{D^{2}+m^{2}}(s)\right|_{s=0},
$$

i.e., $\frac{1}{2}$ the effective action corresponding to the massive Laplacian on the spinor. Because of the $1 / \Gamma(s)$ factor in front of the integral, the simple poles at $s=0$ of the second integral in Eq. (4.1) give rise to a nonvanishing result. Since the singular behavior occurs when $t$ is near 0 (the integral is regular near $t=\infty$ ), we can reduce the integration region to the finite interval $(0,1)$ and use the asymptotic expansion for $\operatorname{Tr}\left[\exp \left(-t \mathbb{D}^{2}\right)\right]$ : 


$$
\operatorname{Tr}\left[\exp \left(-t \mathbb{D}^{2}\right)\right]=\sum_{n=0}^{\infty} H_{n} t^{(n-d) / 2}
$$

to evaluate the integral. Here $H_{n}$ are the Seeley-de Witt coefficients, local functionals of the gauge field and background geometry, invariant under small and large transformations. The integral turns out to be

$$
\begin{gathered}
\mp \frac{\pi i}{2} \zeta_{D^{2}+m^{2}}(0)+\frac{1}{2} \lim _{s \rightarrow 0} \frac{1}{\Gamma(s)} \int_{0}^{1} d t t^{s-1} \\
\times \sum_{n=0}^{\infty} H_{n} t^{(n-d) / 2}\left[\frac{d K^{(1)}}{d s} \pm 2 m \sqrt{t} \frac{d K^{(2)}}{d s}\right] .
\end{gathered}
$$

Let us drop, for the moment, the contribution proportional to $\zeta_{D^{2}+m^{2}}(0)$. Taylor expanding $K^{(i)}$ in the second term and performing the integral in $t$, we obtain for the rest of Eq. (A4)

$$
\begin{gathered}
\frac{1}{2} \lim _{s \rightarrow 0} \sum_{n=0}^{\infty} \sum_{k=0}^{\infty}\left[\frac{H_{n}}{k !} \frac{d^{k}}{d t^{k}}\left(\frac{d}{d s} K^{(1)}(t, s)\right)_{t=0}\right. \\
\quad \times \frac{1}{\Gamma(s)(s+(n-d) / 2+k)} \\
\pm 2 m \frac{H_{n}}{k !} \frac{d^{k}}{d t^{k}}\left(\frac{d}{d s} K^{(2)}(t, s)\right)_{t=0} \\
\left.\quad \times \frac{1}{\Gamma(s)(s+(n-d+1) / 2+k)}\right] .
\end{gathered}
$$

Letting $s \rightarrow 0$ in the previous equation, because of the $1 / \Gamma(s)$, we will get a vanishing result unless $n=d-2 k$ or $n=(d-1)-2 k$. Thus we can write this contribution as

$$
\frac{1}{2} \sum_{n=0}^{\infty} \sum_{k=0}^{\infty}\left[\delta_{n, d-2 k} \frac{H_{n}}{k !} \frac{d^{k}}{d t^{k}}\left(\frac{d}{d s} K^{(1)}(t, s)\right) \pm m \delta_{n,(d-1)-2 k} \frac{H_{n}}{k !} \frac{d^{k}}{d t^{k}}\left(\frac{d}{d s} K^{(2)}(t, s)\right)\right]_{s, t=0} .
$$

Taking account of the fact that only the even coefficient $H_{2 n}$ are different from zero in the heat-kernel expansion for the Laplacian, the first term contributes only if $d$ is even while the second only if $d$ is odd. Explicitly we have

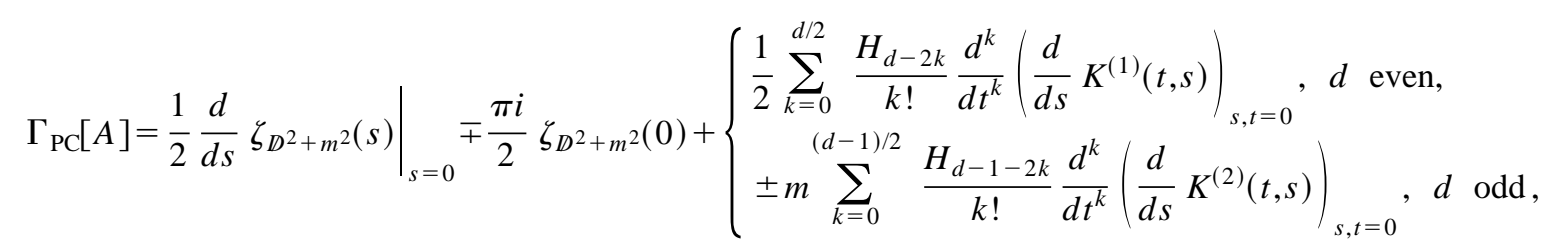

where we have restored the contribution proportional to the $\zeta(0)=\zeta_{D^{2}+m^{2}}(0)$. Let us notice that the cut ambiguity affects odd dimensions, while the local terms in even dimension are insensitive to it. The $\zeta(0)$ part can, as usual, be reabsorbed in a redefinition of the scale. The local parts can be explicitly computed with the result

$$
\begin{aligned}
\Gamma_{\text {loc }}^{\text {odd }}[A]= & \pm \operatorname{sgn}(m) \sqrt{\pi} \sum_{k=0}^{(d-1) / 2} \frac{(-2)^{k}}{(2 k+1) ! !} \\
& \times\left(m^{2}\right)^{k+1 / 2} H_{d-1-2 k}
\end{aligned}
$$

in the odd-dimensional case, while for $d$ even

$$
\begin{aligned}
\Gamma_{\operatorname{loc}}^{\mathrm{even}}[A]= & -\sum_{k=1}^{d / 2}\left[\sum_{j=1}^{k} \frac{(-2)^{j-1}}{j} \frac{1}{(2 j-1) ! !(k-j) !}\right] \\
& \times(i m)^{2 k} H_{d-2 k}
\end{aligned}
$$

\section{APPENDIX B}

There is a very standard technique for evaluating the derivative at $s=0$ of a series such as

$$
\mathcal{F}(s)=\sum_{\mu_{k}} \sum_{n \in \mathbb{Z}}\left[\frac{\beta^{2}}{4 \pi^{2}}\left(m^{2}+\mu_{k}^{2}\right)+\left(a+n+\frac{1}{2}\right)^{2}\right]^{-s}
$$

One starts by writing a Mellin representation of the complex power and then interchanges the sum with the integral in $t$

$$
\begin{aligned}
\mathcal{F}(s)= & \sum_{\mu_{k}} \sum_{n \in \mathbb{Z}} \frac{1}{\Gamma(s)} \int_{0}^{\infty} d t t^{s-1} \\
& \times \exp \left[-\frac{\beta^{2}}{4 \pi^{2}}\left(m^{2}+\mu_{k}^{2}\right) t-\left(a+n+\frac{1}{2}\right)^{2} t\right] \\
= & \frac{1}{\Gamma(s)} \sum_{\mu_{k}} \int_{0}^{\infty} d t t^{s-1} \sum_{n \in \mathbb{Z}} \\
& \times \exp \left[-\frac{\beta^{2}}{4 \pi^{2}}\left(m^{2}+\mu_{k}^{2}\right) t-\left(a+n+\frac{1}{2}\right)^{2} t\right]
\end{aligned}
$$

The above integral exhibits a singularity at $s=0$ when $t$ approaches zero as well. In order to remove this obstacle and thus compute $\mathcal{F}^{\prime}(0)$, we can use Poisson resummation, namely, the identity 


$$
\sum_{n \in Z} f(n)=\sum_{n \in Z} \hat{f}(n)
$$

where $\hat{f}(n) \equiv \int_{-\infty}^{\infty} d x \exp (2 \pi i n x) f(x)$. In our case we have

$$
\begin{aligned}
\mathcal{F}(s)= & \frac{\sqrt{\pi}}{\Gamma(s)} \sum_{\mu_{k}} \int_{0}^{\infty} d t t^{s-3 / 2} \\
& \times \sum_{n \in Z} \exp \left[-\frac{\beta^{2}}{4 \pi^{2}}\left(m^{2}+\mu_{k}^{2}\right) t\right. \\
& \left.-\frac{\pi^{2} n^{2}}{t}-2 \pi i\left(a+\frac{1}{2}\right) n\right] .
\end{aligned}
$$

Notice that the integral is now regular at $t=0$ for every $s$, when $n$ is different from zero, so we can write

$$
\begin{aligned}
\mathcal{F}(s)= & \frac{\sqrt{\pi}}{\Gamma(s)} \sum_{\mu_{k}} \int_{0}^{\infty} d t t^{s-3 / 2} \\
& \times \sum_{n \in \mathbb{Z}, n \neq 0} \exp \left[-\frac{\beta^{2}}{4 \pi^{2}}\left(m^{2}+\mu_{k}^{2}\right) t\right. \\
& \left.-\frac{\pi^{2} n^{2}}{t}-2 \pi i\left(a+\frac{1}{2}\right) n\right] \\
& +\sqrt{\pi} \Gamma(s-1 / 2)\left[\zeta_{\beta^{2} / 4 \pi^{2}\left(\mathbb{D}^{2}+m^{2}\right)}(s-1 / 2)\right. \\
& \left.-\left(\beta^{2} m^{2} / 4 \pi^{2}\right)^{-s+1 / 2}\left(\nu_{+}+\nu_{-}\right)\right] .
\end{aligned}
$$

Performing the integral, we obtain

$$
\begin{aligned}
\mathcal{F}(s)= & \frac{\sqrt{\pi}}{\Gamma(s)} \sum_{\mu_{k}} \sum_{n \in \mathbb{Z}, n \neq 0} \\
& \times \exp \left[-2 \pi i\left(a+\frac{1}{2}\right) n\right] \\
& \times\left(\frac{2 \pi^{2}|n|}{\beta \sqrt{\mu_{k}^{2}+m^{2}}}\right)^{s-1 / 2} K_{s-1 / 2}\left(\beta|n| \sqrt{\mu_{k}^{2}+m^{2}}\right) \\
& +\Gamma(s-1 / 2) \sqrt{\pi}\left[\zeta_{\beta^{2} / 4 \pi^{2}\left(\mathbb{D}^{2}+m^{2}\right)}(s-1 / 2)\right. \\
& \left.-\left(\beta^{2} m^{2} / 4 \pi^{2}\right)^{-s+1 / 2}\left(\nu_{+}+\nu_{-}\right)\right],
\end{aligned}
$$

where $K_{\nu}(x)$ is the Bessel function. We can now take the derivative and let $s \rightarrow 0$, because the series is convergent and defines a holomorphic function at $s=0$,

$$
\begin{aligned}
\mathcal{F}^{\prime}(0)= & \sum_{n \in \mathbb{Z}, n \neq 0} \frac{1}{|n|} \\
& \times \exp \left[-2 \pi i\left(a+\frac{1}{2}\right) n-\beta|n| \sqrt{\mu_{k}^{2}+m^{2}}\right] \\
& +2 \pi \zeta_{\left(\mathbb{D}^{2}+m^{2}\right)}(-1 / 2)-\beta m\left(\nu_{+}+\nu_{-}\right) .
\end{aligned}
$$

Recalling that $\ln (1-x)=\sum_{k=1}^{\infty} x^{k} / k$, we can compute the sum and finally find

$$
\begin{aligned}
\mathcal{F}^{\prime}(0)= & \ln \prod_{\mu_{k}}\left|1+\exp \left[2 \pi i a+\beta \sqrt{\mu_{k}^{2}+m^{2}}\right]\right|^{2} \\
& +2 \pi \zeta_{D^{2}+m^{2}}(-1 / 2)-\beta m\left(\nu_{+}+\nu_{-}\right),
\end{aligned}
$$

as reported in Ref. [6].
[1] S. Deser, L. Griguolo, and D. Seminara, "Finite temperature nonAbelian effective actions," Report No. BRX-TH-426.

[2] W. Siegel, Nucl. Phys. B156, 135 (1979); J. F. Schonfeld, ibid. B185, 157 (1981).

[3] S. Deser, R. Jackiw, and S. Templeton, Phys. Rev. Lett. 48, 975 (1982); Ann. Phys. (N.Y.) 140, 372 (1982).

[4] J. D. Lykken, J. Sonnenschein, and N. Weiss, Int. J. Mod. Phys. A 6, 5155 (1991).

[5] R. Jackiw, Topological Investigations of Quantized Gauge Theories in Current Algebra and Anomalies (Princeton University Press, Princeton, 1985), p. 211; R. Efraty and V. P. Nair, Phys. Rev. Lett. 68, 2891 (1992); R. Jackiw and V. P. Nair, Phys. Rev. D 48, 4991 (1993).

[6] S. Deser, L. Griguolo, and D. Seminara, Phys. Rev. Lett. 79, 1976 (1997).

[7] L. Alvarez-Gaumé, S. Della Pietra, and G. Moore, Ann. Phys. (N.Y.) 163, 288 (1985); A. Niemi, Phys. Rev. Lett. 57, 1102 (1986).

[8] M. Nakahara, Geometry, Topology and Physics (IOP, London, 1990), Chap. 11.

[9] O. Alvarez, Commun. Math. Phys. 100, 279 (1985).

[10] A. Polychronakos, Nucl. Phys. B281, 241 (1987).
[11] R. Dijkgraaf and E. Witten, Commun. Math. Phys. 129, 393 (1990).

[12] S. Deser, L. Griguolo, and D. Seminara, "Definition of ChernSimons terms in $\mathrm{QED}_{3}$ revisited,' Report No. BRX-TH-425, hep-th/9712132 [Commun. Math. Phys. (to be published)].

[13] K. Babu, A. Das, and P. Panigrahi, Phys. Rev. D 36, 3725 (1987); I. Aitchison and J. Zuk, Ann. Phys. (N.Y.) 242, 77 (1995).

[14] R. Pisarski, Phys. Rev. D 35, 664 (1987).

[15] N. Bralić, C. Fosco, and F. Schaposnik, Phys. Lett. B 383, 199 (1996); D. Cabra, E. Fradkin, G. Rossini, and F. Schaposnik, ibid. 383, 434 (1996).

[16] G. Dunne, K. Lee, and C. Lu, Phys. Rev. Lett. 78, 3434 (1997).

[17] R. T. Seeley, Amer. Math. Soc. Proc. Symp. Pure Math. 10, 288 (1967); B. S. DeWitt, Dynamical Theory of Groups and Fields (Gordon and Breach, New York, 1965).

[18] P. B. Gilkey, Invariance Theory, the Heat-Equation and the Atiyah-Singer Index Theorem (CRC, Boca Raton, 1995).

[19] A. N. Redlich, Phys. Rev. Lett. 52, 18 (1984); Phys. Rev. D 29, 2366 (1984).

[20] L. Alvarez-Gaumé and E. Witten, Nucl. Phys. B324, 269 (1983). 
[21] E. Witten, Phys. Lett. 117B, 324 (1982).

[22] O. Aharony, A. Hanany, K. Intriligator, N. Seiberg, and M. J. Strassler, Nucl. Phys. B499, 67 (1997); J. de Boer, K. Hori, and Y. Oz, ibid. B500, 163 (1997).

[23] S. W. Hawking, Commun. Math. Phys. 55, 133 (1977).

[24] R. E. Gamboa Saravi, G. L. Rossini, and F. A. Schaposnik, Int. J. Mod. Phys. A 11, 2643 (1996).

[25] J. Gegelia, A. Khelashvili, and N. Kiknadze, Phys. Rev. D 55, 3897 (1997).

[26] H. Leutwyler, Helv. Phys. Acta 63, 660 (1990).

[27] M. J. Ablowitz and A. S. Fokas, Complex Variables: Introduc- tions and Applications (Cambridge University Press, Cambridge, England, 1997).

[28] I. Affleck, J. A. Harvey, and E. Witten, Nucl. Phys. B206, 413 (1982).

[29] I. S. Gradshteyn and I. M. Ryzhik, Table of Integrals, Series, and Products (Academic, New York, 1980).

[30] C. D. Fosco, G. L. Rossini, and F. A. Schaposnik (a) hep-th/9705124 version 1; (b) hep-th/9705124 version 2; (c) Phys. Rev. Lett. 79, 1980 (1997); (d) 79, 9296(E) (1997).

[31] I. J. R. Aitchison and C. D. Fosco, Phys. Rev. D 57, 1171 (1998). 